present trial might make it the most accurate to date, casting doubt on the validity of such recommendations.

Rachael Williams

Original article Clark WF et al. (2005) Plasma exchange when myeloma presents as acute renal failure: a randomized, controlled trial. Ann Intern Med 143: 777-784

\section{Treatment of malignant glioma with high-dose conformal radiotherapy}

Patients with malignant glioma are usually treated with postoperative chemoradiotherapy; however, tumors often recur at the primary location. Improvements in radiation technology aim to minimize damage to surrounding healthy tissue, which might enable higher doses of radiation to be administered without an increase in the risk of cognitive impairment or necrosis. To investigate the effect of radiation dose on tumor recurrence, Tanaka et al. compared the outcomes of malignant glioma patients treated with conventional radiotherapy with those treated with high-dose conformal radiotherapy.

Patients treated with high-dose radiotherapy (80-90 Gy) within the years 19902002 ( $n=90 ; 29$ with anaplastic astrocytoma and 61 with glioblastoma) were compared with patients treated with conventional radiotherapy (60 Gy) within the years 1979-1989 ( $n=94 ; 34$ with anaplastic astrocytoma and 60 with glioblastoma). After a median followup of 12 months, treatment with high-dose radiotherapy resulted in significantly longer patient survival than conventional radiotherapy (adjusted hazard ratio for death 0.30, 95\% Cl 0.12 to $0.76, P=0.011$ for anaplastic astrocytoma; and adjusted hazard ratio 0.49 , 95\% $\mathrm{Cl} 0.28$ to $0.87, P=0.014$ for glioblastoma). High-dose radiotherapy also increased the duration of progression-free survival in patients with anaplastic astrocytoma, but not in those with glioblastoma. White matter abnormalities were more common in the high-dose group than in the low-dose group $(P=0.0002)$, but toxic effects were generally tolerable.

In summary, compared with conventional 60 Gy radiotherapy, high-dose 80-90 Gy conformal radiotherapy improved survival. Patients treated with high-dose radiotherapy were less likely to have tumor recurrence at the primary site, indicating that higher doses of radiation might be more effective at suppressing tumor regrowth.

Kate Matthews

Original article Tanaka M et al. (2006) High-dose conformal radiotherapy for supratentorial malignant glioma: a historical comparison. Lancet Oncol 6: 953-960

\section{No association between dietary-fiber intake and colorectal cancer}

The hypothesized relationship between dietary-fiber intake and the risk of colorectal cancer has been the focus of numerous epidemiological studies, but the findings have been inconsistent. A recent extensive analysis of 13 international prospective cohort studies, as part of the Pooling Project of Prospective Studies of Diet and Cancer, has shown that there is no significant association between high dietary-fiber intake and a reduced risk of colorectal cancer.

At baseline, study participants $(n=725,628)$ completed questionnaires on food frequency and other nondietary risk factors for colorectal cancer. Over follow-ups ranging between 6 and 20 years, a total of 8,081 cases of colorectal cancer (in 2,776 men and 5,305 women) were recorded. The median energy-adjusted fiber intake ranged from 14 to $28 \mathrm{~g} /$ day in men and 13 to $24 \mathrm{~g} /$ day in women. In the initial age-adjusted analysis, those in the highest fiber-intake quintile had a significantly lower risk (16\%) of developing colorectal cancer than those in the lowest quintile $(P=0.002)$. This inverse association was reduced, but remained significant $(P=0.01)$, when the analysis was adjusted for energy expenditure, nondietary risk factors, and multivitamin use. In the final multivariate model, however, which was adjusted to included folate intake and total meat, milk, and alcohol consumption, the relationship was no longer significant. The authors conclude that this study does not support a significant inverse relationship between dietary-fiber intake and colorectal cancer.

Alexandra King

Original article Park Y et al. (2005) Dietary fiber intake and risk of colorectal cancer: a pooled analysis of prospective cohort studies. JAMA 294: 2849-2857 